\title{
THE KLINE SPHERE CHARACTERIZATION PROBLEM
}

\author{
R. H. BING
}

The object of this paper is to give a solution to the following problem proposed by J. R. Kline: Is a nondegenerate, locally connected, compact continuum which is separated by each of its simple closed curves but by no pair of its points homeomorphic with the surface of a sphere? The answer is in the affirmative.

A solution to the Kline problem gives a characterization of a simple closed surface. Partial solutions of this problem have been made by Hall $[1,2]^{1}$ and Jones [3]. Other characterizations of a simple closed surface have been given by Kuratowski [4], Zippin [5, 6], Wilder [7] and Claytor [8]. Previous to the giving of these characterizations, Moore gave [9] two sets of axioms, each set of which characterized a set topologically equivalent to a plane.

Definition. We say that $M$ disrupts $X$ from $Y$ in $D$ if there is an arc from $X$ to $Y$ in $D$ but each such arc contains a point of $M$.

We shall make use of the following lemma.

Lemma. Suppose that space is locally connected and cannot be separated by the omission of any pair of its points, that the boundary of the connected domain $D$ is equal to the sum of the mutually exclusive sets $M, N$ and $E$, each of which contains a point which is accessible from $D$, and that $D^{\prime}$ is a connected subdomain of $D$ such that no point of $D$ either disrupts $D^{\prime}$ from $E+M$ in $D+E+M$ or disrupts $D^{\prime}$ from $E+N$ in $D+E+N$. Then there is an open arc from $M$ to $N$ in $D$ that does not disrupt $D^{\prime}$ from $E$ in $D+E$.

Proof. Consider the arc $A B$ in $D+B$ from a point $A$ of $D^{\prime}$ to a point $B$ of $E$. Let $W_{1}$ be the set of all points $P$ of $A B$ such that there is an open arc from $P$ to $E$ in $D$ that does not intersect some open arc from $M$ to $N$ in $D$. Assume that the first point $R$ of $A B$ in the order from $A$ to $B$ on the closure of $W_{1}$ does not belong to $D^{\prime}$.

If $R$ disrupts $D^{\prime}$ from $E$ in $D+E$, there are an arc from $D^{\prime}$ to $M$ in $D+M-R$ and an arc from $D^{\prime}$ to $N$ in $D+N-R$. In the sum of these two arcs plus $D^{\prime}$ there is an open arc from $M$ to $N$ in $D$ which does not intersect $R B$. This is contrary to the definition of $R$. Hence, $R$ does not disrupt $D^{\prime}$ from $E$ in $D+E$.

Let $A^{\prime} B^{\prime}$ be an arc in $D+B^{\prime}-R$ from a point $A^{\prime}$ of $D^{\prime}$ to a point

Presented to the Society, November 23, 1945; received by the editors December 3, 1945.

${ }^{1}$ Numbers in brackets refer to the references cited at the end of the paper. 
$B^{\prime}$ of $E$. Since $D^{\prime}$ is connected and does not contain $R$, we may suppose that $A^{\prime}$ is $A$. Let $X$ be the last point of $A^{\prime} B^{\prime}$ in the order from $A^{\prime}$ to $B^{\prime}$ on $A R$ and let $Y$ be a point of $A B$ between $R$ and $B$ such that $R Y$ contains no point of $X B^{\prime}$.

Choose a metric for $X B^{\prime}+X Y$. Let $P_{1}$ be a point of $W_{1} \cdot X Y$ such that no point of $W_{1}$ at a distance of more than 1 from $P_{1}$ is between $P_{1}$ and $X$ on $X Y$. Denote by $V_{1}$ the set of all points $P$ of $X B^{\prime}$ such that there are in $D$ an open arc from $P$ to $E$ and an open arc from $X P_{1}$ to $E$ such that the sum of these two open arcs does not intersect some open arc from $M$ to $N$ in $D$. Let $Q_{1}$ be a point of $V_{1}$ such that no point of $V_{1}$ at a distance of more than 1 from $Q_{1}$ is between $Q_{1}$ and $X$ on $X B^{\prime}$.

Denote by $W_{2}$ the set of all points $P$ of $X Y$ such that there are in $D$ an open arc from $P$ to $E$ and an open arc from $X Q_{1}$ to $E$ such that the sum of these open arcs does not intersect some open arc from $M$ to $N$ in $D$. Let $P_{2}$ be a point of $W_{2} \cdot X P_{1}$ such that no point of $W_{2}$ at a distance of more than $1 / 2$ from $P_{2}$ is between $P_{2}$ and $X$ on $X Y$.

In general, let $W_{n}$ (or $V_{n}$ ) be the set of all points $P$ of $X Y$ (or $X B^{\prime}$ ) such that there are an open arc from $P$ to $E$ in $D$ and an open arc from $X Q_{n-1}$ (or $X P_{n}$ ) to $E$ in $D$ such that the sum of these open arcs does not intersect some open arc from $M$ to $N$ in $D$. Let $P_{n}$ (or $Q_{n}$ ) be a point of $W_{n}$ (or $V_{n}$ ) on $X P_{n-1}$ (or $X Q_{n-1}$ ) such that no point of $W_{n}$ (or $V_{n}$ ) at a distance of more than $1 / n$ from $P_{n}$ (or $Q_{n}$ ) is between $P_{n}\left(\right.$ or $\left.Q_{n}\right)$ and $X$ on $X Y$ (or $\left.X B^{\prime}\right)$.

Let $P_{0}$ and $Q_{0}$ be the limit points of $P_{1}, P_{2}, \cdots$ and $Q_{1}, Q_{2}, \cdots$ respectively. We note that for no integer $n$ is $P_{n}$ between $X$ and $P_{0}$ on $X Y$ or is $Q_{n}$ between $X$ and $Q_{0}$ on $X B^{\prime}$.

Since no pair of points separates space, there is an arc from $A$ to $E$ in space that contains neither $P_{0}$ nor $Q_{0}$. Let $A^{\prime \prime} B^{\prime \prime}$ be a subset of this arc irreducible from $A X+X P_{0}+X Q_{0}$ to $E$. We shall suppose that $A^{\prime \prime}$ is a point of $A X+X P_{0}$ since the argument to follow may be adjusted to take care of the case where it belongs to $A X+X Q_{0}$.

There is an integer $i$ such that $A A^{\prime \prime}$ contains no point of $W_{i}$ and $A^{\prime \prime} B^{\prime \prime}$ intersects neither $P_{0} P_{i}$ nor $Q_{0} Q_{i}$. There is an arc $M^{\prime} N^{\prime}$ in $D+M^{\prime}+N^{\prime}$ from a point $M^{\prime}$ of $M$ to a point $N^{\prime}$ of $N$ such that there are open arcs $\left\langle P^{\prime} B_{1}\right\rangle$ and $\left\langle Q^{\prime} B_{2}\right\rangle$ in $D-\left\langle M^{\prime} N^{\prime}\right\rangle$, where $P^{\prime}$ and $Q^{\prime}$ are points of $P_{0} P_{i}$ and $Q_{0} Q_{i}$ respectively and $B_{j}(j=1,2)$ is a point of $E$. Denote by $P^{\prime \prime}$ the first point of $X P^{\prime}$ in the order from $X$ to $P^{\prime}$ on $P^{\prime} B_{1}+Q^{\prime} B_{2}$ and denote by $Q^{\prime \prime}$ the first point of $X Q^{\prime}$ in the order from $X$ to $Q^{\prime}$ on $P^{\prime} B_{1}+Q^{\prime} B_{2}$. Let $P^{\prime \prime} B_{3}$ and $Q^{\prime \prime} B_{4}$ be arcs in $P^{\prime} B_{1}+Q^{\prime} B_{2}$ where $B_{3}$ and $B_{4}$ are points of $B_{1}+B_{2}$.

Denote the set $A X+X P^{\prime \prime}+X Q^{\prime \prime}$ by $T$. Since no point of $A X$ is a 
point of $W_{i}, P^{\prime \prime} B_{3}+Q^{\prime \prime} B_{4}$ does not intersect $A X$. Then $P^{\prime \prime} B_{3}+Q^{\prime \prime} B_{4}$ intersects $T$ only at $P^{\prime \prime}+Q^{\prime \prime}$.

Now $M^{\prime} N^{\prime}$ intersects $T$ or else there is an open arc from $A$ to $E$ in $T+P^{\prime \prime} B_{3}$ and $R$ is not as assumed. Also, $M^{\prime} N^{\prime}$ does not intersect $T$ in only one point, for if it did, there would be an open arc from $X$ to $E$ in $T+P^{\prime \prime} B_{3}+Q^{\prime \prime} B_{4}$ which would not intersect $M^{\prime} N^{\prime}$. But $X$ is not a point of $W_{i}$.

Let $M^{\prime \prime}$ and $N^{\prime \prime}$ be the first and last points of $M^{\prime} N^{\prime}$ in the order from $M^{\prime}$ to $N^{\prime}$ on $T$. Replace the part of $M^{\prime} N^{\prime}$ between $M^{\prime \prime}$ and $N^{\prime \prime}$ by an arc $\alpha$ from $M^{\prime \prime}$ to $N^{\prime \prime}$ in $T$. Now one of the points $M^{\prime \prime}, N^{\prime \prime}$ belongs to $X P^{\prime \prime}$, for if neither were a point of $X P^{\prime \prime}$, there would be an open arc from $X$ to $E$ in $X P^{\prime \prime}+P^{\prime \prime} B_{3}$ which would not intersect $M^{\prime} M^{\prime \prime}+\alpha+N^{\prime \prime} N^{\prime}$. This contradicts the fact that $X$ is not a point of $W_{i}$. Also, one of $M^{\prime \prime}, N^{\prime \prime}$ is a point of $X Q^{\prime \prime}$. Since $A^{\prime \prime}$ is not a point of $W_{i}$, it is not between $P^{\prime \prime}$ and $M^{\prime \prime}+N^{\prime \prime}$ on $X P^{\prime \prime}$.

Let $C$ be the first point of $A^{\prime \prime} B^{\prime \prime}$ in the order from $A^{\prime \prime}$ to $B^{\prime \prime}$ on $M+M^{\prime} M^{\prime \prime}+N+N^{\prime \prime} N^{\prime}+E+P^{\prime \prime} B_{3}+Q^{\prime \prime} B_{4}$. Now $C$ is not a point of $E+P^{\prime \prime} B_{3}+Q^{\prime \prime} B_{4}$, for if it were, there would be an open arc from $A^{\prime \prime}$ to $E$ in $A^{\prime \prime} C+P^{\prime \prime} B_{3}+Q^{\prime \prime} B_{4}$ that would not intersect $M^{\prime} M^{\prime \prime}+\alpha$ $+N^{\prime \prime} N^{\prime}$. But $A^{\prime \prime}$ is not a point of $W_{i}$.

Assume that $C$ is a point of $M+M^{\prime} M^{\prime \prime}$. Consider an $\operatorname{arc} \beta$ from $M$ to $N$ in $A^{\prime \prime} C+M^{\prime} M^{\prime \prime}+T+N^{\prime \prime} N^{\prime}$ containing $A^{\prime \prime} C$. We note that $\beta$ does not contain $M^{\prime \prime}$. Now $A^{\prime \prime}$ is not a point of $X P^{\prime \prime}$, for if it were, there would be an open arc from $A^{\prime \prime}$ to $E$ in $P^{\prime \prime} B_{3}+Q^{\prime \prime} B_{4}+T$ which would not intersect $\beta$. Also, $A^{\prime \prime}$ is not a point of $A X$ or else there would be an open arc from $X$ to $E$ in $P^{\prime \prime} B_{3}+Q^{\prime \prime} B_{4}+T$ which would not intersect $\beta$. But $X$ is not a point of $W_{i}$. Hence, $C$ is not a point of $M+M^{\prime} M^{\prime \prime}$. Likewise, we find that $C$ is not a point of $N+N^{\prime \prime} N^{\prime}$.

This establishes the lemma. The assumption that $R$ is not a point of $D^{\prime}$ leads to the contradiction that the point $C$ of $M+M^{\prime} M^{\prime \prime}+N$ $+N^{\prime \prime} N^{\prime}+E+P^{\prime \prime} B_{3}+Q^{\prime \prime} B_{4}$ belongs to neither $E+P^{\prime \prime} B_{3}+Q^{\prime \prime} B_{4}$, $M+M^{\prime} M^{\prime \prime}$ nor $N+N^{\prime \prime} N^{\prime}$.

THEOREM. Suppose that $S$ is a nondegenerate, compact continuous curve such that no pair of points but every simple closed curve of $S$ separates it. Then $S$ is topologically equivalent to the surface of a sphere.

Proof. Regard $S$ as space. It is known $[6,10]$ that $S$ is a simple closed surface if no arc separates it. We shall show that no arc separates $S$.

Before giving the details of the proof, we shall briefly outline what we intend to do. On the assumption that some arc separates space, we shall get a finite collection $H_{1}$ of connected domains such that 
their sum does not separate space and such that the sum of any two nonintersecting elements of $H_{1}$ separates the sum of the elements of $H_{1}$. Collections $H_{2}, H_{3}, \cdots$ are defined which satisfy corresponding conditions and which are such that the closure of each element of $H_{n+1}$ is a subset of the sum of the elements of $H_{n}$. See the figure. There

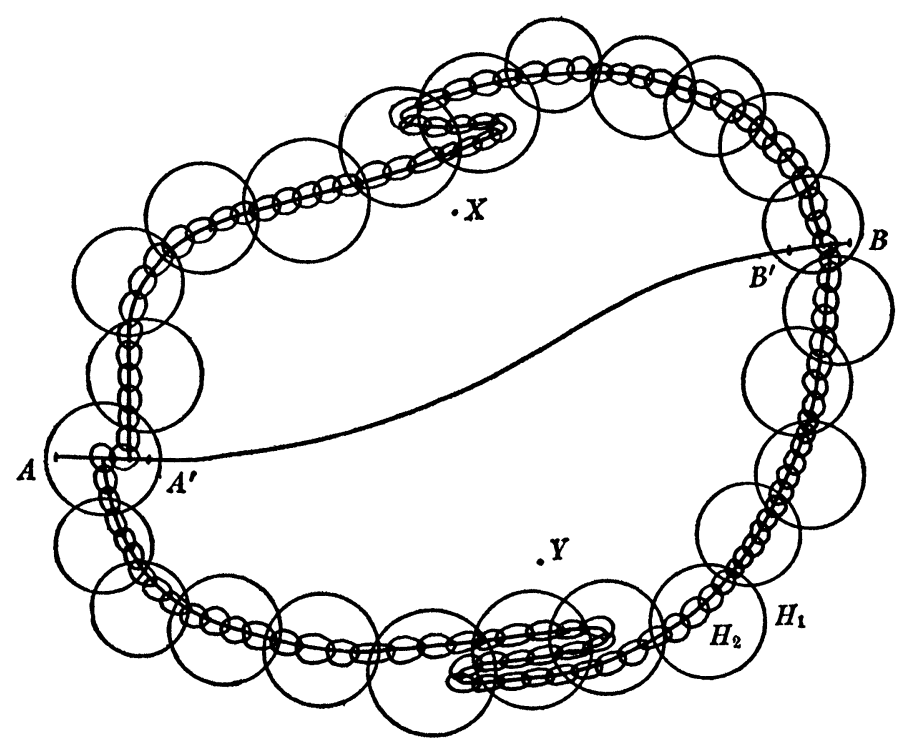

FIG. 1

are actually many more elements in $H_{1}$ and $H_{2}$ than are shown in the figure. The collections $H_{1}, H_{2}, \cdots$ are described in such a way that the common part of their sums is a simple closed curve not separating space. Hence, we shall show that the assumption that some arc separates space leads to the contradiction that some simple closed curve does not. We now consider the details of the proof.

Assume that an arc separates the point $X$ from the point $Y$. Then there is an arc $A B$ that separates $X$ from $Y$ such that no proper subarc of $A B$ separates $X$ from $Y$. Let $D_{X}$ and $D_{Y}$ be the complementary domains of $A B$ containing $X$ and $Y$ respectively.

Description of collection $H_{1}$. Assume that a metric has been chosen for $S$ and let $\epsilon_{1}$ be a positive number less than one one-hundredth of the distance from $A$ to $B$. We shall describe a collection $H_{1}$ of connected domains. The sum of the elements of $H_{1}$ will be denoted by $H_{1}^{*}$. 
The collection $H_{1}$ of connected domains $h_{1,1}, h_{1,2}, \cdots, h_{1, t}(t>100)$ will satisfy the following conditions:

(1) $h_{1, i}$ intersects $h_{1, j}$ only if $i$ is equal to either $j-1, j$ or $j+1$ $\left(h_{1, t+1} \equiv h_{1,1}\right.$ and $\left.h_{1,1-1} \equiv h_{1, t}\right)$;

(2) $S-H_{1}^{*}$ is connected;

(3) some point of $S-H_{1}^{*}$ is accessible from $h_{1,2}$;

(4) the diameter of $h_{1, i}$ is less than $\epsilon_{1}$;

(5) no connected subset of $H_{1}^{*}$ that intersects $h_{1, i}$ and $h_{1, i+2}$ is of diameter less than $\epsilon_{1} / 4$.

Denote by $D_{1}, D_{2}, \cdots, D_{n}$ the elements of a finite collection of connected domains covering $S$ such that the diameter of each is less than $\epsilon_{1} / 100$. Suppose that each of the domains $D_{1}, D_{2}, \cdots, D_{j}$ intersects the complement of $D_{X}+D_{Y}$, each of the domains $D_{j+1}, D_{j+2}, \cdots, D_{k}$ is a subset of $D_{X}$ and each of the domains $D_{k+1}, D_{k+2}, \cdots, D_{n}$ is a subset of $D_{Y}$.

Let $\alpha_{1}, \alpha_{2}, \cdots, \alpha_{j}$ be a collection of arcs in the complement of $D_{X}+D_{Y}+A+B$ such that $\alpha_{i}(i=1, \cdots, j)$ intersects $D_{i}$ and $A B$.

Let $A^{\prime}$ and $B^{\prime}$ be points of the arc $A B$ such that $A A^{\prime}$ and $B^{\prime} B$ are arcs that do not intersect $\alpha_{1}+\alpha_{2}+\cdots+\alpha_{j}$, each of the $\operatorname{arcs} A A^{\prime}$ and $B^{\prime} B$ is covered by an element of $D_{1}, D_{2}, \ldots, D_{n}$, some point of $A^{\prime} B^{\prime}$ is accessible from $D_{Z}(Z=X, Y)$ and if $D_{p}$ is an element of $D_{1}, D_{2}, \cdots, D_{n}$ in $D_{Z}$, then no point of $D_{Z}$ either disrupts $D_{p}$ from $A B^{\prime}$ in $D_{z}+A B^{\prime}$ or disrupts $D_{p}$ from $A^{\prime} B$ in $D_{z}+A^{\prime} B$.

Considering $D_{X}, D_{j+1}, \bar{D}_{X} \cdot\left(A A^{\prime}-A^{\prime}\right), \bar{D}_{X} \cdot\left(B^{\prime} B-B^{\prime}\right)$ and $\bar{D}_{X} \cdot A^{\prime} B^{\prime}$ as $D, D^{\prime}, M, N$ and $E$ of the preceding lemma, we find that there is an arc $\alpha_{j+1}$ from $A^{\prime} B^{\prime}$ to $D_{j+1}$ in $D_{X}+A^{\prime} B^{\prime}$ that does not disrupt $A A^{\prime}-A^{\prime}$ from $B^{\prime} B-B^{\prime}$ in $D_{x}+A B-A^{\prime} B^{\prime}$. Let $D^{\prime}$ be a component of $D_{X}-D_{X} \cdot \alpha_{j+1}$ that contains an open arc from a point of $A A^{\prime}-A^{\prime}$ to a point of $B^{\prime} B-B^{\prime}$. If $D_{j+2}$ is not a subset of $D^{\prime}$, let $\alpha_{j+2}$ be an arc in $D_{X}-D^{\prime}+A^{\prime} B^{\prime}$ from $A^{\prime} B^{\prime}$ to a point of $D_{j+2}$. If $D_{j+2}$ is a subset of $D^{\prime}$, we shall apply the lemma to get an arc $\alpha_{j+2}$ from $D_{j+2}$ to $A^{\prime} B^{\prime}$ in $D_{\mathbf{X}}+A^{\prime} B^{\prime}$ such that $\alpha_{j+1}+\alpha_{j+2}$ does not disrupt $A A^{\prime}-A^{\prime}$ from $B^{\prime} B-B^{\prime}$ in $D_{x}+A B-A^{\prime} B^{\prime}$. The procedure is described in the following paragraph.

Let $R$ be a point of $D^{\prime}$. Since no point of $D_{X}$ disrupts $D_{j+2}$ from $A B^{\prime}$ in $D_{X}+A B^{\prime}$, there is an $\operatorname{arc} \beta$ from $D_{j+2}$ to $A B^{\prime}$ in $D_{X}+A B^{\prime}-R$. A subarc of $\beta$ in $D^{\prime}+A B^{\prime}+\alpha_{j+1}-R$ intersects $D_{j+2}$ and $A B^{\prime}+\alpha_{j+1}$. Hence, $R$ does not disrupt $D_{j+2}$ from $A B^{\prime}+\alpha_{j+1}$ in $D^{\prime}+\bar{D}^{\prime} \cdot\left(A B^{\prime}+\alpha_{j+1}\right)$. Also, $R$ does not disrupt $D_{j+2}$ from $A^{\prime} B+\alpha_{j+1}$ in $D^{\prime}+\bar{D}^{\prime} \cdot\left(A^{\prime} B+\alpha_{j+1}\right)$. Applying the lemma, we find that there is an arc from $D_{j+2}$ to $A^{\prime} B^{\prime}+\alpha_{j+1}$ in $D^{\prime}+A^{\prime} B^{\prime}+\alpha_{j+1}$ that does not disrupt $A A^{\prime}-A^{\prime}$ from $B^{\prime} B-B^{\prime}$ in $D^{\prime}+A B-A^{\prime} B^{\prime}$. Then there is an arc $\alpha_{j+2}$ in $D_{X}+A^{\prime} B^{\prime}$ 
from $D_{j+2}$ to $A^{\prime} B^{\prime}$ such that $\alpha_{j+1}+\alpha_{j+2}$ does not disrupt $A A^{\prime}-A^{\prime}$ from $B^{\prime} B-B^{\prime}$ in $D_{X}+A B-A^{\prime} B^{\prime}$.

Likewise, we find that there is an arc $\alpha_{j+3}$ from $D_{j+3}$ to $A^{\prime} B^{\prime}$ in $D_{X}+A^{\prime} B^{\prime}$ such that $\alpha_{j+1}+\alpha_{j+2}+\alpha_{j+3}$ does not disrupt $A A^{\prime}-A^{\prime}$ from $B^{\prime} B-B^{\prime}$ in $D_{X}+A B-A^{\prime} B^{\prime}$. A continuation of this process gives that there are $\operatorname{arcs} \alpha_{j+1}, \alpha_{j+2}, \cdots, \alpha_{k}$ in $D_{X}+A^{\prime} B^{\prime}$ whose sum does not disrupt $A A^{\prime}-A^{\prime}$ from $B^{\prime} B-B^{\prime}$ in $D_{X}+A B-A^{\prime} B^{\prime}$ and such that $\alpha_{p}(p=i+1, \cdots, k)$ intersects $D_{p}$ and $A^{\prime} B^{\prime}$. Also, there are arcs $\alpha_{k+1}, \alpha_{k+2}, \cdots, \alpha_{n}$ in $D_{Y}+A^{\prime} B^{\prime}$ whose sum does not disrupt $A A^{\prime}-A^{\prime}$ from $B^{\prime} B-B^{\prime}$ in $D_{Y}+A B-A^{\prime} B^{\prime}$ and such that $\alpha_{p}(p=k+1, \cdots, n)$ intersects $D_{p}$ and $A^{\prime} B^{\prime}$.

Let $G$ be the collection of all domains $g$ such that $g$ is a component of the common part of the complement of $A B+\alpha_{1}+\alpha_{2}+\cdots+\alpha_{n}$ and some domain of $D_{1}, D_{2}, \cdots, D_{n}$. If $P$ is a point of $D_{i}$, there is an arc in $D_{i}$ from $P$ to $\alpha_{i}$. Hence, if $g$ is an element of $G$, some point of $A B+\alpha_{1}+\alpha_{2}+\cdots+\alpha_{n}$ is accessible from $g$.

Let $g_{A}$ and $g_{B}$ be domains of diameters less than $\epsilon_{1} / 100$ that cover $A A^{\prime}-A^{\prime}$ and $B^{\prime} B-B^{\prime}$ respectively but no point of $A^{\prime} B^{\prime}+\alpha_{1}+\alpha_{2}$ $+\cdots+\alpha_{n}$. There exists a finite collection $G_{X}$ of domains of $G$ such that this collection but no collection of fewer elements of $G$ satisfies the condition that the sum of the elements of $G_{X}$ is a connected subset of $D_{X}$ and intersects both $g_{A}$ and $g_{B}$. Denote the elements of $G_{X}$ by $g_{2}, g_{3}, \cdots, g_{r}$, where $g_{A}$ intersects $g_{2}, g_{i}(i=2, \cdots, r-1)$ intersects $g_{i+1}$ and $g_{r}$ intersects $g_{B}$. Denote $g_{A}$ by $g_{1}$ and $g_{B}$ by $g_{r+1}$.

Also, there exists a finite collection $G_{Y}$ of domains of $G$ such that this collection but no collection of fewer elements of $G$ satisfies the condition that the sum of the elements of $G_{Y}$ is a connected subset of $D_{Y}$ and intersects both $g_{A}$ and $g_{B}$. Denote the elements of $G_{Y}$ by $g_{r+2}, g_{r+3}, \cdots, g_{s}$ where $g\left(i_{i}=r+1, \cdots, s-1\right)$ intersects $g_{i+1}$ and $g_{s}$ intersects $g_{1}$.

Let $E$ denote $g_{1}+g_{2}+\cdots+g_{s}$ plus all points that it separates from $A^{\prime} B^{\prime}$. Each component of the common part of $E$ and an element of $G$ intersects an element of $g_{1}, g_{2}, \cdots, g_{s}$. However, it is to be noted that no such component intersects two $g_{i}$ 's that do not belong to a consecutive set of three domains of $g_{1}, g_{2}, \cdots, g_{8}, g_{1}, g_{2}$. Denote by $g_{i}^{\prime}$ the sum of $g_{i}$ and all such components that intersect $g_{i}$. We note that $g_{i}^{\prime}$ is a diameter less than $\epsilon_{1} / 33$.

If three is a factor of $s$, denote the sum of the first three elements of $g_{1}^{\prime}, g_{2}^{\prime}, \cdots, g_{s}^{\prime}$ by $h_{1}$, the sum of the next three elements by $h_{2}, \cdots$ and the sum of the last three elements by $h_{v}$. If three is a factor of $s+1$, then $h_{1}, h_{2}, \cdots$ and $h_{v}$ are defined as before except that $h_{v}$ is the sum of the last four elements of $g_{1}^{\prime}, g_{2}^{\prime}, \ldots, g_{s}^{\prime}$ instead 
of the last three. If three is a factor of $s+2$, each of $h_{v-1}$ and $h_{v}$ is the sum of four elements of $g_{1}^{\prime}, g_{2}^{\prime}, \cdots, g_{s}^{\prime}$. Since each $h_{i}$ contains either $g_{A}, g_{B}$ or an element $g$ of $G$ which does not intersect $g_{A}+g_{B}$ and since a point of $A B+\alpha_{1}+\alpha_{2}+\cdots+\alpha_{n}$ is accessible from $g$, then a point of $A^{\prime} B^{\prime}+\alpha_{1}+\alpha_{2}+\cdots+\alpha_{n}$ is accessible from $h_{i}$. Now $h_{i}$ is of diameter less than $\epsilon_{1} / 8$ and the collection $h_{1}, h_{2}, \cdots, h_{v}$ satisfies conditions analogous to conditions (1), (2) and (3) to be satisfied by $h_{1,1}, h_{1,2}, \cdots, h_{1, t}$.

Let $h_{1,1}$ be the sum of $h_{1}, h_{2}, \cdots, h_{n}$ where some connected subset of $h_{1}+h_{2}+\cdots+h_{v}$ of diameter less, than $\epsilon_{1} / 4$ intersects $h_{1}, h_{2}, \cdots$ and $h_{n}$ but no such subset intersects both $h_{1}$ and $h_{n+1}$; let $h_{1,2}$ be the sum of $h_{n+1}, h_{n+2}, \cdots, h_{m}$ where some connected subset of $h_{1}+h_{2}+\cdots+h_{v}$ of diameter less than $\epsilon_{1} / 4$ intersects $h_{n+1}, h_{n+2}, \cdots$ and $h_{m}$ but no such subset intersects both $h_{n+1}$ and $h_{m+1} ; \cdots$; and let $h_{1, t}$ be the sum of $h_{p+1}, h_{p+2}, \cdots, h_{v}$ where some connected subset of $h_{1}+h_{2}+\cdots+h_{v}$ of diameter less than $3 \epsilon_{1} / 4$ intersects $h_{p+1}$, $h_{p+2}, \cdots$ and $h_{v}$ but no subset of $h_{1}+h_{2}+\cdots+h_{v}$ of diameter less than $\epsilon_{1} / 4$ intersects both $h_{p+1}$ and $h_{1}$. We use $3 \epsilon_{1} / 4$ in the last case instead of $\epsilon_{1} / 4$ in order to insure that no connected set in $h_{1}+h_{2}+\cdots+h_{v}$ of diameter less than $\epsilon_{1} / 4$ intersects both $h_{p+1}$ and $h_{1}$. The collection $H_{1}$ satisfies conditions (1), (2), (3), (4) and (5).

Description of collection $\mathrm{H}_{2}$. Choose a positive number $\epsilon_{2}$ less than one one-hundredth of the diameter of any connected set in $H_{1}^{*}$ that intersects $h_{1, i}$ and $h_{1, i+2}$. We shall describe a collection $H_{2}$ of connected domains $h_{2,1}, h_{2,2}, \cdots, h_{2,8}$ such that:

(1) $h_{2, i}$ intersects $h_{2, j}$ only if $i$ is equal to either $j-1, j$ or $j+1$ $\left(h_{2,8+1} \equiv h_{2,1}\right.$ and $\left.h_{2,1-1} \equiv h_{2, s}\right)$;

(2) $\mathrm{S}-\mathrm{H}_{2}{ }^{*}$ is connected;

(3) some point of $S-H_{2}{ }^{*}$ is accessible from $h_{2, i}$;

(4) the diameter of $h_{2, i}$ is less than $\epsilon_{2}$;

(5) no connected subset of $H_{2}{ }^{*}$ that intersects $h_{2, i}$ and $h_{2, i+2}$ is of diameter less than $\epsilon_{2} / 4$;

(6) each $h_{1, i}$ contains 98 consecutive elements of $h_{2,1}, h_{2,2}, \cdots, h_{2,8}$;

(7) if $H(n ; i, j)$ denotes $h_{n, i-100}+\cdots+h_{n, i}+\cdots+h_{n, j}+\cdots$ $+h_{n, j+100}$ and $h_{1, i}$ and $h_{1, j}$ intersect $h_{2, m}$ and $h_{2, k}$ respectively, then either $H(1 ; i, j)$ covers the closure of $H(2 ; m, k)$ and $H(1 ; j, i)$ covers the closure of $H(2 ; k, m)$ or $H(1 ; i, j)$ covers the closure of $H(2 ; k, m)$ and $H(1 ; j, i)$ covers the closure of $H(2 ; m, k)$.

Denote by $C$ the component that contains $h_{1,5}+h_{1,6}$ of the common part of $h_{1,2}+h_{1,3}+\cdots+h_{1,9}$ and the complement of the closure of $h_{1,1}+h_{1,10}$. We shall show that if $P$ is a point of $h_{1,5}+h_{1,6}$ and $R$ is a 
point of $C-P$, then $R$ does not disrupt $P$ from $S-H_{1}^{*}$ in $S-H_{1}^{*}+C$. Let $P Q$ be an arc in $S-R$ from $P$ to a point $Q$ of $S-H_{1}^{*}$. Let $Q^{\prime}$ be the first point of $P Q$ in the order from $P$ to $Q$ on $S-C$. If $P Q^{\prime}$ intersects $h_{1,3}$, then there is an arc from $P Q^{\prime}-Q^{\prime}$ to $S-H_{1}^{*}$ in $S-H_{1}^{*}+h_{1,3}$ because a point of $S-H_{1}^{*}$ is accessible from $h_{1,3}$. Also, if $P Q^{\prime}$ intersects $h_{1,8}, R$ does not disrupt $P$ from $S-H_{1}^{*}$ in $S-H_{1}^{*}+C$. If $P Q^{\prime}$ intersects neither $h_{1,3}$ nor $h_{1,8}$, then $Q^{\prime}$ is a point of $S-H_{1}^{*}$. This demonstrates that $R$ does not disrupt $P$ from $S-H_{1}^{*}$ in $S-H_{1}^{*}+C$.

Let $G$ be a finite collection of connected domains covering $h_{1,5}+h_{1,6}$ such that each intersects $h_{1,5}+h_{1,6}$ and is of diameter less than $\epsilon_{2} / 1200$. No point of $C$ disrupts an element of $G$ from $S-H_{1}^{*}$ in $S-H_{1}^{*}+C$. Repeated applications of the preceding lemma give that there is a continuum $K$ in $S-H_{1}^{*}+C$ that intersects $A^{\prime} B^{\prime}$ and each element of $G$ but does not disrupt $h_{1,1}$ from $h_{1,10}$ in $h_{1,1}+h_{1,2}+\cdots$ $+h_{1,10}$. Let $G^{\prime}$ be the set of all domains $g^{\prime}$ such that $g^{\prime}$ is either the common part of $h_{1,2}+h_{1,3}+\cdots+h_{1,9}$, the complement of $K$ and an element of $G$, or the common part of the complement of $K$ and $h_{i}$ for $i$ equal to $2,3,4,7,8$ or 9 .

There exists a finite collection $G^{\prime \prime}$ of elements of $G^{\prime}$ such that the sum of the elements of $G^{\prime \prime}$ is a connected domain intersecting $h_{1,1}$ and $h_{1,10}$ but the sum of no subcollection of $G^{\prime}$ having fewer elements than $G^{\prime \prime}$ is a connected domain intersecting $h_{1,1}$ and $h_{1,10}$. Assume that $g_{1}$ of $G^{\prime \prime}$ intersects $h_{1,1}, g_{i}(i=1, \cdots, r-1)$ intersects $g_{i+1}$ and $g_{r}$ intersects $h_{1,10}$.

There exists a collection $g_{1}^{\prime}, g_{2}^{\prime}, \cdots, g_{r}^{\prime}$ of connected domains such that $g_{1}^{\prime}$ intersects $h_{1,1}, g_{i}^{\prime}$ intersects $g_{i+1}^{\prime}, g_{r}^{\prime}$ intersects $h_{1,10}$ and the closure of $g_{k}^{\prime}(k=1, \cdots, r)$ is a subset of $g_{k}$.

Let $E$ denote $h_{1,1}+g_{1}^{\prime}+\cdots+g_{r}^{\prime}+h_{1,10}+\cdots+h_{1, t}$ plus all points that it separates from $A^{\prime} B^{\prime}$. Each component of the common part of $E$ and an element of $G^{\prime}$ intersects one of the domains $h_{1,1}$, $g_{1}{ }^{\prime}, \cdots, g_{r}^{\prime}, h_{1,10}$ but no such component intersects two of these domains that do not belong to a consecutive set of three of these domains. Add such components to the ones of $h_{1,1}, g_{i}^{\prime}, h_{1,10}$ that they intersect to form the sets $h_{1}, g_{i}^{\prime \prime}, h_{10}$. We note that the diameter of each $g_{i}^{\prime \prime}$ not intersecting $h_{1,2}+h_{1,3}+h_{1,4}+h_{1,7}+h_{1,8}+h_{1,9}$ is less than $\epsilon_{2} / 400$.

Consecutive elements of $g_{1}^{\prime \prime}, g_{2}^{\prime \prime}, \cdots, g_{r}^{\prime \prime}$ may be combined by threes and fours in a manner previously described so as to get a collection $g_{1,1}, g_{1,2}, \cdots, g_{1, u}$ such that the collection $h_{1}, g_{1,1}, \cdots$, $g_{1, u}, h_{10}, h_{1,11}, \cdots, h_{1, t}$ satisfies conditions analogous to conditions (1), (2) and (3) to be satisfied by $h_{2,1}, h_{2,2}, \cdots, h_{2,8}$. We note that the closure of $g_{1, i}(2 \leqq i<u)$ is a subset of $h_{1,1}+h_{1,2}+\cdots+h_{1,10}$. 
In a manner similar to that in which $h_{1,1}+h_{1,2}+\cdots+h_{1,10}$ was replaced by $h_{1}+g_{1,1}+\cdots+g_{1, u}+h_{10}$, we replace $h_{1,11}+\cdots+h_{1,20}$ by $h_{11}+g_{11,1}+\cdots+g_{11, v}+h_{20}, \cdots$ and $h_{1, t-m}+\cdots+h_{t}(9 \leqq m$ $\leqq 18)$ by $h_{t-m}+g_{t-m, 1}+\cdots+g_{t-m, w}+h_{t}$.

Let $g_{i, 0}$ be the fourth element of $g_{i, 1}, g_{i, 2}, \cdots, g_{i, n}$ which follows all of these elements that intersect $h_{1, i+3}$. We note that $g_{i, 0}$, the three domains immediately preceding $g_{i, 0}$, and the three domains immediately following $g_{i, 0}$ are each a subset of $h_{1, i+4}$ of diameter less than $\epsilon_{2} / 100$.

In the manner described above, replace $g_{1,0}+\cdots+h_{10}+h_{11}+\cdots$ $+g_{11,0}$ by $g_{1,0}^{\prime}+h_{2,2}^{\prime}+\cdots+h_{2, r}^{\prime}+g_{11,0}^{\prime}$; replace $g_{11,0}^{\prime}+\cdots+g_{21,0}$ by $h_{2, r+1}^{\prime}+\cdots+h_{2, v}^{\prime}+g_{21,0}^{\prime} ; \cdots$ and replace $g_{t-m, 0}^{\prime}+\cdots+g_{1,0}^{\prime}$ by $h_{2, n}^{\prime}+\cdots+h_{2, u}^{\prime}+h_{2,1}^{\prime}$. The closure of $h_{2,2}^{\prime}+\cdots+h_{2, r}^{\prime}$ is a subset of $h_{1,4}+\cdots+h_{1,20}, \cdots$ and the closure of $h_{2, n}^{\prime}+\cdots+h_{2, u}^{\prime}+h_{2,1}^{\prime}$ is a subset of $h_{1, t-m+3}+\cdots+h_{1,10}$. Consecutive elements of $h_{2,1}^{\prime}, h_{2,2}^{\prime}$, ..., $h_{2, u}^{\prime}$ may be combined in a manner previously described so as to form a collection $H_{2}$ of connected domains $h_{2,1}, h_{2,2}, \cdots, h_{2,8}$ satisfying conditions (1), (2), (3), (4), (5), (6) and (7).

Description of simple closed curve $J$. For each positive integer $i$ greater than one, we define a collection $H_{i}$ of connected domains $h_{i, 1}, h_{i, 2}, \cdots, h_{i, n}$ satisfying conditions analogous to those satisfied by $H_{2}$ where $\epsilon_{i}$ is a positive number less than one one-hundredth the diameter of any connected set in $H_{i-1}^{*}$ intersecting $h_{i-1, j}$ and $h_{i-1, j+2}$. We shall show that the common part $J$ of $H_{1}^{*}, H_{2}^{*}, \ldots$ is a simple closed curve that does not separate $S$.

As the closure of $H_{i+1}{ }^{*}$ is a connected subset of $H_{i}{ }^{*}$ (condition 7) and as each $h_{i, j}$ contains an element of $H_{i+1}$ (condition 6), then $J$ is a nondegenerate continuum. This continuum does not separate space because the complement of each $H_{i}{ }^{*}$ is connected.

To show that $J$ is a simple closed curve, we shall show that any pair of points $P, Q$ of $J$ separates it. Suppose that $h_{i, P_{i}}$ and $h_{i, Q_{i}}$ are elements of $h_{i, 1}, \cdots, h_{i, n}$ that contain $P$ and $Q$ respectively. For convenience in notation, we shall assume that it is $H\left(i ; P_{i}, Q_{i}\right)$ that covers the closure of $H\left(i+1 ; P_{i+1}, Q_{i+1}\right)$ and that it is $H\left(i ; Q_{i}, P_{i}\right)$ that covers the closure of $H\left(i+1 ; Q_{i+1}, P_{i+1}\right)$. If $J_{P Q}$ is the common part of $H\left(1 ; P_{1}, Q_{1}\right), H\left(2 ; P_{2}, Q_{2}\right), \cdots$, we find that $J$ is the sum of two continua $J_{P Q}$ and $J_{Q P}$ which have only $P$ and $Q$ in common.

Hence, the assumption that an arc separates $S$ leads to the conclusion that some simple closed curve does not. 


\section{REFERENCES}

1. D. W. Hall, A partial solution of a problem of J. R. Kline, Duke Math. J. vol. 9 (1942) pp. 893-901.

2. —, A note on primitive skew curves, Bull. Amer. Math. Soc. vol. 49 (1943) pp. $935-936$.

3. F. B. Jones, Bull. Amer. Math. Soc. Abstract 48-11-340.

4. C. Kuratowski, Une caracterisation topologique de la surface de la sphère, Fund. Math. vol. 13 (1929) pp. 307-318.

5. L. Zippin, $A$ study of continuous curves and their relation to the JaniszewskiMullikin Theorem, Trans. Amer. Math. Soc. vol. 31 (1929) pp. 744-770.

6. - On continuous curves and the Jordan Curve Theorem, Amer. J. Math. vol. 52 (1930) pp. 331-350.

7. R. L. Wilder, A converse of the Jordan-Brouwer separation theorem in three dimensions, Trans. Amer. Math. Soc. vol. 32 (1930) pp. 632-651.

8. S. Claytor, Topological immersion of Peanian continua in a spherical surface, Ann. of Math. vol. 35 (1934) pp. 809-835.

9. R. L. Moore, Concerning a set of postulates for plane analysis situs, Trans. Amer. Math. Soc. vol. 20 (1919) pp. 169-178.

10. E. R. van Kampen, On some characterizations of 2-dimensional manifolds, Duke Math. J. vol. 1 (1935) pp. 74-93.

The UnIversity of TeXas 